\title{
A Modification Fractional Homotopy Perturbation Method for Solving Helmholtz and Coupled Helmholtz Equations on Cantor Sets
}

\author{
Dumitru Baleanu ${ }^{1,2}$ (D) and Hassan Kamil Jassim ${ }^{3, *(D)}$ \\ 1 Department of Mathematics, Faculty of Art and Sciences, Çankaya University, Ankara 06530, Turkey; \\ dumitru@cankaya.edu.tr \\ 2 Institute of Space Sciences, Magurele-Bucharest 077125, Romania \\ 3 Department of Mathematics, Faculty of Education for Pure Sciences, University of Thi-Qar, \\ Nasiriyah 64001, Iraq \\ * Correspondence: hassan.kamil@yahoo.com
}

Received: 12 May 2019; Accepted: 31 May 2019; Published: 3 June 2019

\begin{abstract}
In this paper, we apply a new technique, namely, the local fractional Laplace homotopy perturbation method (LFLHPM), on Helmholtz and coupled Helmholtz equations to obtain analytical approximate solutions. The iteration procedure is based on local fractional derivative operators (LFDOs). This method is a combination of the local fractional Laplace transform (LFLT) and the homotopy perturbation method (HPM). The method in general is easy to implement and yields good results. Illustrative examples are included to demonstrate the validity and applicability of the new technique.
\end{abstract}

Keywords: Helmholtz equation; local fractional homotopy perturbation method; local fractional Laplace transform; local fractional derivative operator

\section{Introduction}

The theory of local fractional calculus was successfully utilized to describe the non-differentiable problems arising in mathematical physics, such as Schrödinger equations [1], the gas dynamic equation [2], the telegraph equation [3], the wave equation [4-7], Fokker-Planck equations [8,9], Laplace equations [10], Klein-Gordon equations [11], Helmholtz equations [12], and the Goursat problem [13] on Cantor sets.

Several analytical and numerical methods have been used to solve partial differential equations (PDEs) with local fractional derivative operators (LFDOs), such as the Adomian decomposition method [13-15], variational iteration method [16-22], differential transform method [23,24], series expansion method [25], Sumudu transform method [26], Laplace transform method [27], reduced differential transform method [28], Laplace variational iteration method [29], Fourier series method [30], and homotopy perturbation method [31]. Our aim is to present the coupling method of local fractional Laplace transform (LFLT) and homotopy perturbation method (HPM), which we call the local fractional Laplace homotopy perturbation method (LFLHPM), and use it to solve differential Helmholtz and coupled Helmholtz equations on Cantor sets within a local fractional operator.

This paper is organized as follows. In Section 2, the basic mathematical tools of local fractional calculus are introduced. The analysis of the proposed method is given in Section 3. Then, in Sections 4 and 5 , the proposed method is implemented to solve differential Helmholtz and coupled Helmholtz equations on Cantor sets within a local fractional operator. Finally, concluding remarks are presented in Section 6. 


\section{Mathematical Fundamentals}

Definition 1. The local fractional derivative of $\varphi(\mu)$ of order $\delta$ at the point $\mu_{0}$ is given by $[19,20,32,33]$ :

$$
\varphi^{(\delta)}\left(\mu_{0}\right)=\lim _{\mu \rightarrow \mu_{0}} \frac{\Gamma(1+\delta)\left[\varphi(\mu)-\varphi\left(\mu_{0}\right)\right]}{\left(\mu-\mu_{0}\right)^{\delta}}, 0<\delta \leq 1 .
$$

Definition 2. Let $\frac{1}{\Gamma(1+\delta)} \int_{0}^{\infty}|\varphi(\mu)|(d \mu)^{\delta}<k<\infty$. The local fractional Laplace transform of $\varphi(\mu)$ is given by $[19,20,32,33]$ :

$$
L T_{\delta}\{\varphi(\mu)\}=\Omega(\mu, w)=\frac{1}{\Gamma(1+\delta)} \int_{0}^{\infty} E_{\delta}\left(-w^{\delta} \mu^{\delta}\right) \varphi(\mu)(d \mu)^{\delta},
$$

where the latter integral converges and $w^{\delta} \in R^{\delta}$.

Definition 3. The inverse formula of the local fractional Laplace transform is given by the following:

$$
L T_{\delta}^{-1}[\Omega(\mu, w)]=\varphi(\mu)=\frac{1}{(2 \pi)^{\delta}} \int_{\beta-i \infty}^{\beta+i \infty} E_{\delta}\left(w^{\delta} \mu^{\delta}\right) \Omega(\mu, w)(d w)^{\delta}
$$

where $w^{\delta}=\beta+i \omega ;$ fractal imaginary unit $i^{\delta}$ and $\operatorname{Re}(w)=\beta>0$.

Definition 4. The Mittage-Leffler function and hyperbolic sine are, respectively, defined by $[19,20,32,33]$ :

$$
\begin{aligned}
E_{\delta}\left(\mu^{\delta}\right) & =\sum_{k=0}^{\infty} \frac{\mu^{k \delta}}{\Gamma(1+k \delta)}, \mu \in R, 0<\delta \leq 1, \\
\sinh _{\delta}\left(\mu^{\delta}\right) & =\frac{E_{\delta}\left(\mu^{\delta}\right)-E_{\delta}\left(-\mu^{\delta}\right)}{2}, \mu \in R, 0<\delta \leq 1 .
\end{aligned}
$$

The properties for the local fractional Laplace transform used in the paper are given as follows:

1. $\operatorname{LT}_{\delta}\left\{\varphi^{(k \delta)}(\mu)\right\}=w^{k \delta} L T_{\delta}\{\varphi(\mu)\}-w^{(k-1) \delta} \varphi(0)-\cdots-\varphi^{((k-1) \delta)}(0)$.

2. $L T_{\delta}\left\{\mu^{k \delta}\right\}=\frac{\Gamma(1+k \delta)}{w^{(k+1) \delta}}$.

\section{Analysis of LFLHPM}

The local fractional homotopy perturbation method (LFHPM) has been developed and applied to solve a class of local fractional partial differential equations [31,34]. Based on it, we suggest a new analytical method.

Let us consider the following partial differential equation with local fractional derivative:

$$
L_{\delta} \varphi(\mu, \tau)+R_{\delta} \varphi(\mu, \tau)=f(\mu, \tau), \quad 0<\delta \leq 1
$$

where $L_{\delta}=\frac{\partial^{k \delta}}{\partial \mu^{k \delta}}, R_{\delta}$ is a linear local fractional operator, and $f(\mu, \tau)$ is the source term.

Applying the local fractional Laplace transform (LFLT) to Equation (6), it gives the following:

$$
\Omega(\mu, w)=w^{-\delta} \varphi(0, \tau)+w^{-2 \delta} \varphi^{(\delta)}(0, \tau)+\cdots+w^{-(k-1) \delta} \varphi^{((k-1) \delta)}(0, \tau)+w^{-k \delta} L T_{\delta}\{f(\mu, \tau)\}-w^{-k \delta} L T_{\delta}\left\{R_{\delta} \varphi(\mu, \tau)\right\} .
$$

Using the inversion of LFLT on Equation (7), we have the following:

$$
\varphi(\mu, \tau)=G(\mu, \tau)-\operatorname{LT}_{\delta}^{-1}\left[w^{-k \delta} \operatorname{LT}_{\delta}\left\{R_{\delta} \varphi(\mu, \tau)\right\}\right]
$$


where $G(\mu, \tau)$ represents the term arising from the source term and the prescribed initial conditions. Now, we apply the LFHPM [31]:

$$
\varphi(\mu, \tau)=\sum_{n=0}^{\infty} p^{n} \varphi_{n}(\mu, \tau)
$$

Using Equation (9) in Equation (8), it yields the following result:

$$
\sum_{n=0}^{\infty} p^{n} \varphi_{n}(\mu, \tau)=G(\mu, \tau)-p \operatorname{LT}_{\delta}^{-1}\left[w^{-k \delta} \operatorname{LT}_{\delta}\left\{R_{\delta} \sum_{n=0}^{\infty} p^{n} \varphi_{n}(\mu, \tau)\right\}\right] .
$$

On equating the multipliers of same powers of the parameter $p$ of Equation (10), it gives the following:

$$
\begin{gathered}
p^{0}: \varphi_{0}(\mu, \tau)=G(\mu, \tau), \\
p^{1}: \varphi_{1}(\mu, \tau)=-L T_{\delta}^{-1}\left[w^{-k \delta} L T_{\delta}\left\{R_{\delta} \varphi_{0}(\mu, \tau)\right\}\right], \\
p^{2}: \varphi_{2}(\mu, \tau)=-L T_{\delta}^{-1}\left[w^{-k \delta} L T_{\delta}\left\{R_{\delta} \varphi_{1}(\mu, \tau)\right\}\right], \\
p^{3}: \varphi_{3}(\mu, \tau)=-L T_{\delta}^{-1}\left[w^{-k \delta} L T_{\delta}\left\{R_{\delta} \varphi_{2}(\mu, \tau)\right\}\right],
\end{gathered}
$$

Proceeding in this same manner, the rest of the components $\varphi_{n}(\mu, \tau)$ can be completely obtained and the series solution is thus entirely determined. Finally, we approximate the analytical solution $\varphi(\mu, \tau)$ by truncated series as follows:

$$
\varphi(\mu, \tau)=\lim _{N \rightarrow \infty} \sum_{n=0}^{N} \varphi_{n}(\mu, \tau)
$$

\section{Application of LFLHPM for Helmholtz Equations}

Example 1. Let us consider the local fractional Helmholtz equation with local fractional derivative operator:

$$
\frac{\partial^{2 \delta} \varphi(\mu, \tau)}{\partial \mu^{2 \delta}}+\frac{\partial^{2 \delta} \varphi(\mu, \tau)}{\partial \tau^{2 \delta}}+\varphi(\mu, \tau)=\frac{\mu^{\delta}}{\Gamma(1+\delta)} \frac{\tau^{\delta}}{\Gamma(1+\delta)}, \quad 0<\delta \leq 1
$$

with fractal initial boundary conditions

$$
\varphi(0, \tau)=0, \quad \varphi^{(\delta)}(0, \tau)=\frac{\tau^{\delta}}{\Gamma(1+\delta)} \quad(0<\tau \leq l) .
$$

Applying the LFLT on both sides (12), subject to initial condition (13), we have the following:

$$
\Omega(\mu, w)=w^{-2 \delta} \frac{\tau^{\delta}}{\Gamma(1+\delta)}+w^{-2 \delta} L T_{\delta}\left\{\frac{\mu^{\delta}}{\Gamma(1+\delta)} \frac{\tau^{\delta}}{\Gamma(1+\delta)}\right\}-w^{-2 \delta} L T_{\delta}\left\{\frac{\partial^{2 \delta} \varphi(\mu, \tau)}{\partial \tau^{2 \delta}}+\varphi(\mu, \tau)\right\}
$$

The inversion of LFLT implies that

$$
\varphi(\mu, \tau)=\frac{\mu^{\delta}}{\Gamma(1+\delta)} \frac{\tau^{\delta}}{\Gamma(1+\delta)}+\frac{\mu^{3 \delta}}{\Gamma(1+3 \delta)} \frac{\tau^{\delta}}{\Gamma(1+\delta)}-L T_{\delta}^{-1}\left[w^{-2 \delta} L T_{\delta}\left\{\frac{\partial^{2 \delta} \varphi(\mu, \tau)}{\partial \tau^{2 \delta}}+\varphi(\mu, \tau)\right\}\right] .
$$

Now, applying LFHPM, we obtain the following:

$$
\sum_{n=0}^{\infty} p^{n} \varphi_{n}(\mu, \tau)=\frac{\mu^{\delta}}{\Gamma(1+\delta)} \frac{\tau^{\delta}}{\Gamma(1+\delta)}+\frac{\mu^{3 \delta}}{\Gamma(1+3 \delta)} \frac{\tau^{\delta}}{\Gamma(1+\delta)}-p L T_{\delta}^{-1}\left[w^{-2 \delta} L T_{\delta}\left\{\frac{\partial^{2 \delta}}{\partial \tau^{2 \delta}}\left(\sum_{n=0}^{\infty} p^{n} \varphi_{n}(\mu, \tau)\right)+\sum_{n=0}^{\infty} p^{n} \varphi_{n}(\mu, \tau)\right\}\right] .
$$


Comparing the coefficients of like power of $p$, we get the following:

$$
\begin{gathered}
p^{0}: \varphi_{0}(\mu, \tau)=\frac{\mu^{\delta}}{\Gamma(1+\delta)} \frac{\tau^{\delta}}{\Gamma(1+\delta)}+\frac{\mu^{3 \delta}}{\Gamma(1+3 \delta)} \frac{\tau^{\delta}}{\Gamma(1+\delta)}, \\
p^{1}: \varphi_{1}(\mu, \tau)=-L T_{\delta}^{-1}\left[w^{-2 \delta} L T_{\delta}\left\{\frac{\partial^{2 \delta}}{\partial \tau^{2 \delta}} \varphi_{0}(\mu, \tau)+\varphi_{0}(\mu, \tau)\right\}\right], \\
p^{2}: \varphi_{2}(\mu, \tau)=-L T_{\delta}^{-1}\left[w^{-2 \delta} L T_{\delta}\left\{\frac{\partial^{2 \delta}}{\partial \tau^{2 \delta}} \varphi_{1}(\mu, \tau)+\varphi_{1}(\mu, \tau)\right\}\right], \\
p^{3}: \varphi_{3}(\mu, \tau)=-L T_{\delta}^{-1}\left[w^{-2 \delta} L T_{\delta}\left\{\frac{\partial^{2 \delta}}{\partial \tau^{2 \delta}} \varphi_{2}(\mu, \tau)+\varphi_{2}(\mu, \tau)\right\}\right],
\end{gathered}
$$

Hence, we have:

$$
\begin{gathered}
p^{0}: \varphi_{0}(\mu, \tau)=\frac{\mu^{\delta}}{\Gamma(1+\delta)} \frac{\tau^{\delta}}{\Gamma(1+\delta)}+\frac{\mu^{3 \delta}}{\Gamma(1+3 \delta)} \frac{\tau^{\delta}}{\Gamma(1+\delta)^{3}}, \\
p^{1}: \varphi_{1}(\mu, \tau)=-\frac{\mu^{3 \delta}}{\Gamma(1+3 \delta)} \frac{\tau^{\delta}}{\Gamma(1+\delta)}-\frac{\mu^{5 \delta}}{\Gamma(1+5 \delta)} \frac{\tau^{\delta}}{\Gamma(1+\delta)}, \\
p^{2}: \varphi_{2}(\mu, \tau)=\frac{\mu^{5 \delta}}{\Gamma(1+5 \delta)} \frac{\tau^{\delta}}{\Gamma(1+\delta)}+\frac{\mu^{7 \delta}}{\Gamma(1+7 \delta)} \frac{\tau^{\delta}}{\Gamma(1+\delta)}, \\
p^{3}: \varphi_{3}(\mu, \tau)=-\frac{\mu^{7 \delta}}{\Gamma(1+7 \delta)} \frac{\tau^{\delta}}{\Gamma(1+\delta)}-\frac{\mu^{9 \delta}}{\Gamma(1+9 \delta)} \frac{\tau^{\delta}}{\Gamma(1+\delta)},
\end{gathered}
$$

Therefore, the series solution of Equation (12) is given by the following:

$$
\varphi(\mu, \tau)=\lim _{N \rightarrow \infty} \sum_{n=0}^{N} \varphi_{n}(\mu, \tau)=\frac{\mu^{\delta}}{\Gamma(1+\delta)} \frac{\tau^{\delta}}{\Gamma(1+\delta)} .
$$

The result is the same as the one which is obtained by the local fractional variational iteration method [35].

\section{Application of LFLHPM for Coupled Helmholtz Equations}

Example 2. Let us consider the coupled Helmholtz equations with local fractional derivative operators:

$$
\begin{aligned}
& \frac{\partial^{2 \delta} \varphi(\mu, \tau)}{\partial \mu^{2 \delta}}+\frac{\partial^{2 \delta} \psi(\mu, \tau)}{\partial \tau^{2 \delta}}-\varphi(\mu, \tau)=0, \\
& \frac{\partial^{2 \delta} \psi(\mu, \tau)}{\partial \mu^{2 \delta}}+\frac{\partial^{2 \delta} \varphi(\mu, \tau)}{\partial \tau^{2 \delta}}-\psi(\mu, \tau)=0
\end{aligned}
$$

subject to the initial boundary conditions

$$
\begin{gathered}
\varphi(0, \tau)=0, \varphi^{(\delta)}(0, \tau)=E_{\delta}\left(\tau^{\delta}\right),(0<\tau \leq l) \\
\psi(0, \tau)=0, \psi^{(\delta)}(0, \tau)=-E_{\delta}\left(\tau^{\delta}\right) .(0<\tau \leq l) .
\end{gathered}
$$

Applying the LFLT on both sides (18), subject to initial condition (19), we have the following:

$$
\begin{array}{r}
\Omega(\mu, w)=w^{-2 \delta} E_{\delta}\left(\tau^{\delta}\right)+w^{-2 \delta} L T_{\delta}\left\{\varphi(\mu, \tau)-\frac{\partial^{2 \delta} \psi(\mu, \tau)}{\partial \tau^{2 \delta}}\right\}, \\
\Psi(\mu, w)=-w^{-2 \delta} E_{\delta}\left(\tau^{\delta}\right)+w^{-2 \delta} L T_{\delta}\left\{\psi(\mu, \tau)-\frac{\partial^{2 \delta} \varphi(\mu, \tau)}{\partial \tau^{2 \delta}}\right\} .
\end{array}
$$


The inversion of LFLT implies that

$$
\begin{aligned}
& \varphi(\mu, \tau)=\frac{\mu^{\delta}}{\Gamma(1+\delta)} E_{\delta}\left(\tau^{\delta}\right)+L T_{\delta}^{-1}\left[w^{-2 \delta} L T_{\delta}\left\{\varphi(\mu, \tau)-\frac{\partial^{2 \delta} \psi(\mu, \tau)}{\partial \tau^{2 \delta}}\right\}\right] \\
& \psi(\mu, \tau)=-\frac{\mu^{\delta}}{\Gamma(1+\delta)} E_{\delta}\left(\tau^{\delta}\right)+L T_{\delta}^{-1}\left[w^{-2 \delta} L T_{\delta}\left\{\psi(\mu, \tau)-\frac{\partial^{2 \delta} \varphi(\mu, \tau)}{\partial \tau^{2 \delta}}\right\}\right]
\end{aligned}
$$

Now, applying LFHPM, we obtain the following:

$$
\begin{aligned}
\varphi(\mu, \tau) & =\sum_{n=0}^{\infty} p^{n} \varphi_{n}(\mu, \tau), \\
\psi(\mu, \tau) & =\sum_{n=0}^{\infty} p^{n} \psi_{n}(\mu, \tau) .
\end{aligned}
$$

Using Equation (22) in Equation (21), it yields the following result:

$$
\begin{gathered}
\sum_{n=0}^{\infty} p^{n} \varphi_{n}(\mu, \tau)=\frac{\mu^{\delta}}{\Gamma(1+\delta)} E_{\delta}\left(\tau^{\delta}\right)+p L T_{\delta}^{-1}\left[w^{-2 \delta} L T_{\delta}\left\{\sum_{n=0}^{\infty} p^{n} \varphi_{n}(\mu, \tau)-\frac{\partial^{2 \delta}}{\partial \tau^{2 \delta}}\left(\sum_{n=0}^{\infty} p^{n} \psi_{n}(\mu, \tau)\right)\right\}\right], \\
\sum_{n=0}^{\infty} p^{n} \psi_{n}(\mu, \tau)=-\frac{\mu^{\delta}}{\Gamma(1+\delta)} E_{\delta}\left(\tau^{\delta}\right)+p \operatorname{LT}_{\delta}^{-1}\left[w^{-2 \delta} L T_{\delta}\left\{\sum_{n=0}^{\infty} p^{n} \psi_{n}(\mu, \tau)-\frac{\partial^{2 \delta}}{\partial \tau^{2 \delta}}\left(\sum_{n=0}^{\infty} p^{n} \varphi_{n}(\mu, \tau)\right)\right\}\right] .
\end{gathered}
$$

Comparing the coefficients of like power of $p$, we get the following:

$$
\begin{gathered}
p^{0}:\left\{\begin{array}{l}
\varphi_{0}(\mu, \tau)=\frac{\mu^{\delta}}{\Gamma(1+\delta)} E_{\delta}\left(\tau^{\delta}\right), \\
\psi_{0}(\mu, \tau)=-\frac{\mu^{\delta}}{\Gamma(1+\delta)} E_{\delta}\left(\tau^{\delta}\right),
\end{array}\right. \\
p^{1}:\left\{\begin{array}{l}
\varphi_{1}(\mu, \tau)=L T_{\delta}^{-1}\left[w^{-2 \delta} L T_{\delta}\left\{\varphi_{0}(\mu, \tau)-\frac{\partial^{2 \delta}}{\partial \tau^{2 \delta}} \psi_{0}(\mu, \tau)\right\}\right], \\
\psi_{1}(\mu, \tau)=L T_{\delta}^{-1}\left[w^{-2 \delta} L T_{\delta}\left\{\psi_{0}(\mu, \tau)-\frac{\partial^{2 \delta}}{\partial \tau^{2 \delta}} \varphi_{0}(\mu, \tau)\right\}\right],
\end{array}\right. \\
p^{2}:\left\{\begin{array}{l}
\varphi_{2}(\mu, \tau)=L T_{\delta}^{-1}\left[w^{-2 \delta} L T_{\delta}\left\{\varphi_{1}(\mu, \tau)-\frac{\partial^{2 \delta}}{\partial \tau^{2 \delta}} \psi_{1}(\mu, \tau)\right\}\right], \\
\psi_{2}(\mu, \tau)=L T_{\delta}^{-1}\left[w^{-2 \delta} L T_{\delta}\left\{\psi_{1}(\mu, \tau)-\frac{\partial^{2 \delta}}{\partial \tau^{2 \delta}} \varphi_{1}(\mu, \tau)\right\}\right],
\end{array}\right.
\end{gathered}
$$

Hence, we have:

$$
\begin{aligned}
& p^{0}:\left\{\begin{array}{l}
\varphi_{0}(\mu, \tau)=\frac{\mu^{\delta}}{\Gamma(1+\delta)} E_{\delta}\left(\tau^{\delta}\right), \\
\psi_{0}(\mu, \tau)=-\frac{\mu^{\delta}}{\Gamma(1+\delta)} E_{\delta}\left(\tau^{\delta}\right),
\end{array}\right. \\
& p^{1}:\left\{\begin{array}{l}
\frac{2 \mu^{3 \delta}}{\Gamma(1+3 \delta)} E_{\delta}\left(\tau^{\delta}\right), \\
\varphi_{1}(\mu, \tau)=-\frac{2 \mu^{3 \delta}}{\Gamma(1+3 \delta)} E_{\delta}\left(\tau^{\delta}\right), \\
\psi_{1}(\mu, \tau)
\end{array}\right. \\
& p^{2}:\left\{\begin{array}{l}
\frac{4 \mu^{5 \delta}}{\Gamma(1+5 \delta)} E_{\delta}\left(\tau^{\delta}\right), \\
\varphi_{2}(\mu, \tau)=-\frac{4 \mu^{5 \delta}}{\Gamma(1+5 \delta)} E_{\delta}\left(\tau^{\delta}\right), \\
\psi_{2}(\mu, \tau)=\frac{8 \mu^{7 \delta}}{\Gamma(1+7 \delta)} E_{\delta}\left(\tau^{\delta}\right), \\
\varphi_{2}(\mu, \tau)=-\frac{8 \mu^{7 \delta}}{\Gamma(1+7 \delta)} E_{\delta}\left(\tau^{\delta}\right), \\
\psi_{2}(\mu, \tau)=
\end{array}\right.
\end{aligned}
$$


and so on, and the other components can be found in a similar manner. Therefore, the series solutions can be written in the following form:

$$
\begin{aligned}
\varphi(\mu, \tau) & =\lim _{N \rightarrow \infty} \sum_{n=0}^{N} \varphi_{n}(\mu, \tau) \\
& =E_{\delta}\left(\tau^{\delta}\right)\left[\frac{\mu^{\delta}}{\Gamma(1+\delta)}+\frac{2 \mu^{3 \delta}}{\Gamma(1+\delta)}+\frac{4 \mu^{5 \delta}}{\Gamma(1+\delta)}+\cdots\right] \\
& =E_{\delta}\left(\tau^{\delta}\right) \frac{\sinh \left(\sqrt{2} \mu^{\delta}\right)}{\sqrt{2}} . \\
(\mu, \tau) & =\lim _{N \rightarrow \infty} \sum_{n=0}^{N} \psi_{n}(\mu, \tau) \\
& =-E_{\delta}\left(\tau^{\delta}\right)\left[\frac{\mu^{\delta}}{\Gamma(1+\delta)}+\frac{2 \mu^{3 \delta}}{\Gamma(1+\delta)}+\frac{4 \mu^{5 \delta}}{\Gamma(1+\delta)}+\cdots\right] \\
& =-E_{\delta}\left(\tau^{\delta}\right) \frac{\sinh _{\delta}\left(\sqrt{2} \mu^{\delta}\right)}{\sqrt{2}} .
\end{aligned}
$$

The result is the same as the one which is obtained by the local fractional variational iteration transform method [36].

\section{Conclusions}

In this work, the LFLHPM was successfully applied to finding the approximate solution of Helmholtz and coupled Helmholtz equations involving LFDOs. A comparison was made to show that the method has a small computational size in comparison with the computational size required in other numerical methods, such as the local fractional variational iteration method and the local fractional variational iteration transform method. The method is very powerful and efficient in finding analytical as well as numerical solutions for wide classes of linear and nonlinear local fractional PDEs.

Author Contributions: H.K.J. wrote some sections of the manuscript; D.B. prepared some other sections of the paper and analyzed. All authors have read and approved the final manuscript.

Funding: This research received no external funding.

Conflicts of Interest: The authors declare no conflict of interest

\section{References}

1. Jafari, H.; Jassim, H.K. Local Fractional Series Expansion Method for Solving Laplace and Schrodinger Equations on Cantor Sets within Local Fractional Operators. Int. J. Math. Comput. Res. 2014, 2, 736-744.

2. Baleanu, D.; Jassim, H.K. A Modification Fractional Variational Iteration Method for solving Nonlinear Gas Dynamic and Coupled KdV Equations Involving Local Fractional Operators. Therm. Sci. 2018, 22, S165-S175. [CrossRef]

3. Jafari, H.; Jassim, H.K. Application of the Local Fractional Adomian Decomposition and Series Expansion Methods for Solving Telegraph Equation on Cantor Sets Involving Local Fractional Derivative Operators. J. Zankoy Sulaimani Part A 2015, 17, 15-22. [CrossRef]

4. Jassim, H.K.; Ünlü, C.; Moshokoa, S.; Khalique, M. Local Fractional Laplace Variational Iteration Method for Solving Diffusion and Wave Equations on Cantor Sets within Local Fractional Operators. Math. Probl. Engin. 2015, 2015, 1-9. [CrossRef]

5. Baleanu, D.; Machado, J.A.T.; Cattani, C.; Baleanu, M.C.; Yang, X.-J. Local fractional variational iteration and Decomposition methods for Wave equation on Cantor set within local fractional Operators. Abstr. Appl. Anal. 2014, 2014, 1-6. [CrossRef]

6. Jassim, H.K. The Approximate Solutions of Three-Dimensional Diffusion and Wave Equations within Local Fractional Derivative Operator. Abstr. Appl. Anal. 2016, 2016, 1-5. [CrossRef] 
7. Wang, S.Q.; Yang, Y.; Jassim, H.K. Local Fractional Function Decomposition Method for Solving Inhomogeneous Wave Equations with Local Fractional Derivative. Abstr. Appl. Anal. 2014, 2014, 1-7. [CrossRef]

8. Yang, X.J.; Baleanu, D. Local fractional variational iteration method for Fokker-Planck equation on a Cantor set. Acta Univ. 2013, 23, 3-8.

9. Jassim, H.K. New Approaches for Solving Fokker Planck Equation on Cantor Sets within Local Fractional Operators. J. Math. 2015, 2015, 1-8. [CrossRef]

10. Yan, S.P.; Jafari, H.; Jassim, H.K. Local Fractional Adomian Decomposition and Function Decomposition Methods for Solving Laplace Equation within Local Fractional Operators. Adv. Math. Phys. 2014, 2014, 1-7. [CrossRef]

11. Yang, A.M.; Zhang, Y.Z.; Cattani, C.; Xie, G.N.; Rashidi, M.M.; Zhou, Y.J.; Yang, X.J. Application of local fractional series expansion method to solve Klein-Gordon equations on Cantor sets. Abstr. Appl. Anal. 2014, 2014, 372741. [CrossRef]

12. Jassim, H.K. The Approximate Solutions of Helmholtz and Coupled Helmholtz Equations on Cantor Sets within Local Fractional Operator. J. Zankoy Sulaimani Part A 2015, 17, 19-25. [CrossRef]

13. Baleanu, D.; Jassim, H.K.; Qurashi, M.A. Approximate Analytical Solutions of Goursat Problem within Local Fractional Operators. J. Nonlinear Sci. Appl. 2016, 9, 4829-4837. [CrossRef]

14. Jamshad, A.; Syed, T.M.-D. Local Fractional Decomposition Method on Wave Equation in Fractal Strings. Mitteilungen Klosterneuburg 2014, 64, 98-105.

15. Jafari, H.; Jassim, H.K. Local Fractional Adomian Decomposition Method for Solving Two Dimensional Heat Conduction Equations within Local Fractional Operators. J. Adv. Math. 2014, 9, 2574-2582.

16. Baleanu, D.; Jassim, H.K. Approximate Solutions of the Damped Wave Equation and Dissipative Wave Equation in Fractal Strings. Fractal Fract. 2019, 3, 26. [CrossRef]

17. Jafari, H.; Jassim, H.K. Local Fractional Variational Iteration Method for Nonlinear Partial Differential Equations within Local Fractional Operators. Appl. Appl. Math. 2015, 10, 1055-1065.

18. Xu, S.; Ling, X.; Zhao, Y.; Jassim, H.K. A Novel Schedule for Solving the Two-Dimensional Diffusion Problem in Fractal Heat Transfer. Therm. Sci. 2015, 19, S99-S103. [CrossRef]

19. Yang, X.J. Advanced Local Fractional Calculus and Its Applications; World Science Publisher: New York, NY, USA, 2012.

20. Yang, A.M.; Chen, Z.; Srivastava, H.M.; Yang, X.J. Application of the Local Fractional Series Expansion Method and the Variational Iteration Method to the Helmholtz Equation Involving Local Fractional Derivative Operators. Abstr. Appl. Anal. 2013, 2013, 259125. [CrossRef]

21. Jafari, H.; Jassim, H.K.; Vahidi, J. The Reduced Differential Transform and Variational Iteration Methods for 3D Diffusion Model in Fractal Heat Transfer within Local Fractional Operators. Therm. Sci. 2018, 22, S301-S307. [CrossRef]

22. Jassim, H.K.; Shahab, W.A. Fractional variational iteration method to solve one dimensional second order hyperbolic telegraph equations. J. Phys. Conf. Ser. 2018, 1032, 1-9. [CrossRef]

23. Yang, X.J.; Machad, J.A.; Srivastava, H.M. A new numerical technique for solving the local fractional diffusion equation: Two-dimensional extended differential transform approach. Appl. Math. Comput. 2016, 274, 143-151. [CrossRef]

24. Jafari, H.; Jassim, H.K.; Tchier, F.; Baleanu, D. On the Approximate Solutions of Local Fractional Differential Equations with Local Fractional Operator. Entropy 2016, 18, 150. [CrossRef]

25. Yang, A.M.; Yang, X.J.; Li, Z.B. Local fractional series expansion method for solving wave and diffusion equations Cantor sets. Abstr. Appl. Anal. 2013, 2013,1-5. [CrossRef]

26. Srivastava, H.M.; Golmankhaneh, A.K.; Baleanu, D. Local Fractional Sumudu Transform with Application to IVPs on Cantor Set. Abstr. Appl. Anal. 2014, 2014,1-7. [CrossRef]

27. Zhao, C.G.; Yang, A.M.; Jafari, H.; Haghbin, A. The Yang-Laplace Transform for Solving the IVPs with Local Fractional Derivative. Abstr. Appl. Anal. 2014, 2014, 1-5. [CrossRef]

28. Jafari, H.; Jassim, H.K.; Moshokoa, S.P.; Ariyan, V.M.; Tchier, F. Reduced differential transform method for partial differential equations within local fractional derivative operators. Adv. Mech. Eng. 2016, 6, 1-6. [CrossRef] 
29. Jafari, H.; Jassim, H.K. Local Fractional Laplace Variational Iteration Method for Solving Nonlinear Partial Differential Equations on Cantor Sets within Local Fractional Operators. J. Zankoy Sulaimani Part A 2014, 16, $49-57$.

30. Hu, M.S.; Agarwal, R.P.; Yang, X.J. Local Fractional Fourier Series with Application to Wave Equation in Fractal Vibrating. Abstr. Appl. Anal. 2012, 2012, 1-7. [CrossRef]

31. Zhang, Y.; Cattani, C.; Yang, X.J. Local Fractional Homotopy Perturbation Method for Solving Non-Homogeneous Heat Conduction Equations in Fractal Domains. Entropy 2015, 17, 6753-6764. [CrossRef]

32. Kolwankar, K.M.; Gangal, A.D. Fractional differentiability of nowhere differentiable functions and dimensions. Chaos 1996, 6, 505-513. [CrossRef]

33. Kolwankar, K.M.; Gangal, A.D. Local fractional Fokker-Planck equation. Phys. Rev. Lett. 1998, 80, $214-217$. [CrossRef]

34. Singh, J.; Kumar , D.; Baleanu , D.; Rathore , S.; et al. On the local fractional wave equation in fractal strings. Math. Method Appl. Sci. 2019, 2019, 1-8. [CrossRef]

35. Wang, X.J.; Zhao, Y.; Cattani, C.; Yang, X.J. Local Fractional Variational Iteration Method for Inhomogeneous Helmholtz Equation within Local Fractional Derivative Operator. Math. Probl. Eng. 2014, 2014, 913202. [CrossRef]

36. Yong, J.Y.; Liu, Q.H. Variational Iteration Transform Method for Fractional Differential Equations with Local Fractional Derivative. Abstr. Appl. Anal. 2014, 2014, 760957. [CrossRef]

(C) 2019 by the authors. Licensee MDPI, Basel, Switzerland. This article is an open access article distributed under the terms and conditions of the Creative Commons Attribution (CC BY) license (http://creativecommons.org/licenses/by/4.0/). 